\title{
Hydrolytic enzymes (proteases and lipases) released by biofilm-forming cells of Scedosporium/ Lomentospora species
}

\begin{abstract}
Introduction and objective: Scedosporium/Lomentospora species are human opportunistic filamentous fungi able to form biofilm in both biotic and abiotic surfaces. However, little is known about the ability of these fungi to release molecules into the biofilm environment. In this context, the present study aimed to detect hydrolytic enzymes, proteases and lipases, in the supernatants obtained from biofilm-forming cells of S. apiospermum, S. minutisporum, $S$. aurantiacum and L. prolificans (formerly S. prolificans).
\end{abstract}

Materials and methods: Biofilms were formed through incubation of Scedosporium and Lomentospora conidial cells for 72 hours over a polystyrene surface and validated by classical biomarkers (biomass, extracellular matrix and viability). In parallel, the cell-free biofilm supernatants were harvested in order to measure the activity of secreted hydrolytic enzymes, proteases and lipases. Azoalbumin and azocasein were used as proteinaceous substrates, while 4-methylumbelliferyl butyrate, 4-methylumbelliferyl heptanoate and 4-methylumbelliferyl oleate were used as lipid substrates.

Results: As previously reported, mature biofilms formed by Scedosporium/Lomentospora species were confirmed by the presence of a dense mycelial mass covering the polystyrene surface. Protease and lipase activities were detected in the biofilm-derived supernatants of all fungi studied. Regarding the protease, similar cleavages were observed for both azo-substrates, conjugated to albumin and casein, with an overall predilection to neutralalkaline $\mathrm{pH}$ range. Concerning the lipase, substrates containing lipids with small, medium and large carbonic chains were degraded, with different predilection according to the fungal species.

The inferences: Collectively, the results suggest the presence of distinct proteases and lipases secreted by biofilm-growing cells of $S$. apiospermum, S. minutisporum, $S$. aurantiacum and L. prolificans.

Keywords: Scedosporium, Lomentospora, biofilm, proteases, lipases, secretion
Volume 7 Issue 6 - 2019

\author{
Thaís P Mello,' luri C Barcellos, ,,2 Marta H \\ Branquinha,' André LS Santos ${ }^{1,3}$ \\ 'Laboratório de Estudos Avançados de Microrganismos \\ Emergentes e Resistentes, Departamento de Microbiologia \\ Geral, Instituto de Microbiologia Paulo de Góes, Universidade \\ Federal do Rio de Janeiro, Brazil \\ ${ }^{2}$ Instituto Federal de Educação, Ciência e Tecnologia do Rio de \\ Janeiro (IFRJ), Brazil \\ PPrograma de Pós-Graduação em Bioquímica, Instituto de \\ Química, UFRJ, Brazil
}

\begin{abstract}
Correspondence: André Santos, Laboratório de Estudos Avançados de Microrganismos Emergentes e Resistentes (LEAMER), Departamento de Microbiologia Geral, Instituto de Microbiologia Paulo de Góes (IMPG), Centro de Ciências da Saúde (CCS), Bloco E subsolo, Sala 05, Universidade Federal do Rio de Janeiro (UFRJ), Avenida Carlos Chagas Filho, 373, Rio de Janeiro, 2 194I-902, Brazil, Tel +55 2I 39380366, Email andre@micro.ufrj.br
\end{abstract}

Received: November 08, 2019 | Published: December 17, 2019 


\section{Biofilm assay}

For biofilm formation, conidial suspensions in Sabouraud medium (200 $\mu$ l containing $10^{6}$ cells) were placed on flat-bottom 96-well polystyrene microtiter plates and then incubated without agitation at $37^{\circ} \mathrm{C}$ with $5 \%$ of $\mathrm{CO}_{2}$ for 72 hours. Medium-only blanks were also set up in parallel. ${ }^{3}$ Subsequently, the supernatants were harvested and filtered through a 0.22- $\mu \mathrm{m}$ membrane (Millipore, Brazil). Protein concentration of the cell-free biofilm supernatants was determined by the method using the Folin phenol reagent and bovine serum albumin (BSA) as the protein standard. ${ }^{4}$ In parallel, to confirm the ability of these fungi to form biofilm over polystyrene surface, the biomass was evidenced by crystal violet dye in methanol-fixed biofilm as well as the extracellular matrix and the metabolic activity were quantified in non-fixed biofilm by safranin incorporation and XTT metabolization, respectively. ${ }^{5,6}$

\section{Protease activity}

Protease activity was assayed using two azo-containing proteinaceous substrates, azocasein and azoalbumin (Sigma-Aldrich, USA), at different pH's (4.0, 5.0, 7.0 and 9.0). Briefly, the cell-free supernatants (100 $\mu \mathrm{g}$ of proteins) were incubated with azocasein or azoalbumin $(1.6 \mathrm{mg} / \mathrm{ml})$ in $50 \mathrm{mM}$ sodium acetate buffer $(\mathrm{pH} 4.0)$, $50 \mathrm{mM}$ sodium phosphate buffer $(\mathrm{pH} 5.0$ ), PBS ( $\mathrm{pH} 7.0)$ or 50 $\mathrm{mM}$ glycine- $\mathrm{NaOH}\left(\mathrm{pH} \mathrm{9.0)}\right.$, for 1 hour at $37^{\circ} \mathrm{C}$. The reaction was terminated by adding $5 \%$ trichloroacetic acid. After centrifugation at $4000 \mathrm{~g}$ for 5 minutes, the supernatants were collected and the absorbance of the released azo-dye read at $366 \mathrm{~nm}$ in a microplate reader (SpectraMax M3; Molecular Devices, USA). The specific peptidase activity was expressed in arbitrary units (U) per milligram of protein, where 1 unit of enzymatic activity is equivalent to the variation in optical density of $0.001 \mathrm{~nm}$ per minuteat $366 \mathrm{~nm}^{4}$

\section{Lipase activity}

Lipase activity was assayed using three distinct lipid substrates, 4-methylumbelliferyl butyrate, 4-methylumbelliferyl heptanoate and 4-methylumbelliferyl oleate (Sigma-Aldrich, Brazil). To perform the reaction mixtures, $10 \mu \mathrm{M}$ of each substrate was added with cell-free supernatants (100 $\mu \mathrm{g}$ of proteins) in $10 \mathrm{mM}$ Tris- $\mathrm{HCl}$ buffer ( $\mathrm{pH} 8.0)$, as suggested by the manufacture protocol. Cleavage of substrates was monitored for 1 hour at $37^{\circ} \mathrm{C}$ in a fluorescence spectrometer (SpectraMax Gemini XPS; Molecular Devices, USA) using an excitation wavelength of $327 \mathrm{~nm}$ and an emission wavelength of 449 $\mathrm{nm}$. The lipase activity was expressed as the fluorescence arbitrary units (FAU) obtained after 60 minutes of reaction.

\section{Results}

The Scedosporium/Lomentospora mature biofilms were evidenced by classical methodologies, including the observation of biomass by crystal violet staining, the presence of extracellular polymeric substance by safranin dye and the metabolic activity of biofilmforming cells by XTT metabolization (Figure 1). In addition, light microscopy analyzes clearly revealed a dense fungal mass formed with intertwined mycelial cells (Figure 1). Subsequently, the supernatants of biofilm-growing cells of Scedosporium/Lomentospora were harvested in order to measure the hydrolytic activity of two classes of enzymes, proteases and lipases.

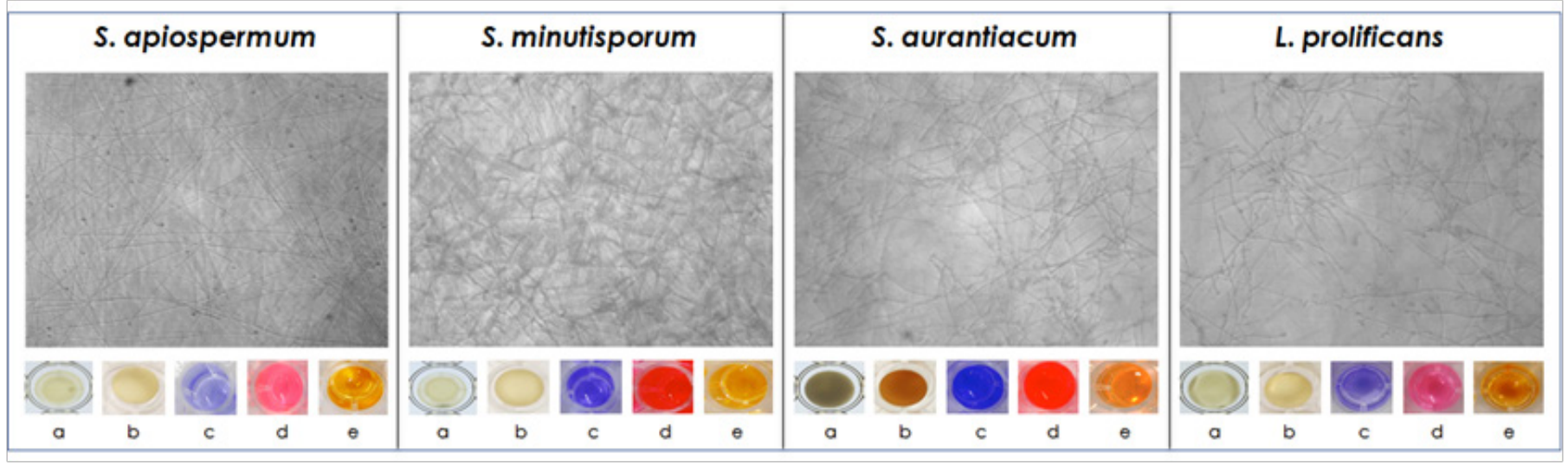

Figure I Light microscopies of biofilms formed by S. apiospermum, S. minutisporum, S. aurantiacum and L. prolificans after 72 hours of interaction with polystyrene surface. In addition, images of the mature biofilms formed on 96-well microtiter polystyrene plate: (a) top and (b) bottom view of the wells, (c) biofilm biomass evidenced by crystal violet, (d) biofilm extracellular matrix evidenced by safranin incorporation and (e) biofilm metabolic activity evidenced by XTT metabolization.

Overall, protease activities secreted by $S$. apiospermum, $S$. minutisporum, S. aurantiacum and L. prolificans were preferentially evidenced at neutral-alkaline $\mathrm{pH}$ range regarding the two proteinaceous substrates tested, albumin and casein (Figure 2). However, considerable differences on preferential degradation of both proteins were evidenced. For instance, in the extreme acidic $\mathrm{pH}$ (4.0), higher protease activities were detected over azocasein than azoalbumin in all fungi. In $\mathrm{pH}$ 5.0, S. apiospermum and S. minutisporum hydrolyzed better azocasein than azoalbumin and an opposite cleavage pattern was detected for S. aurantiacum and L. prolificans (Figure 2).
Lipase activities able to cleave different lipid chain sizes were detected in all fungal species studied herein (Figure 3). For S. apiospermum and L. prolificans, the higher lipase activity was detected over the substrate containing a medium lipid chain size (4-methylumbelliferyl heptanoate), while S. minutisporum preferentially degrades the small lipid size chain (4-methylumbelliferyl butyrate) and $S$. aurantiacum the larger one (4-methylumbelliferyl oleate) (Figure 3). 


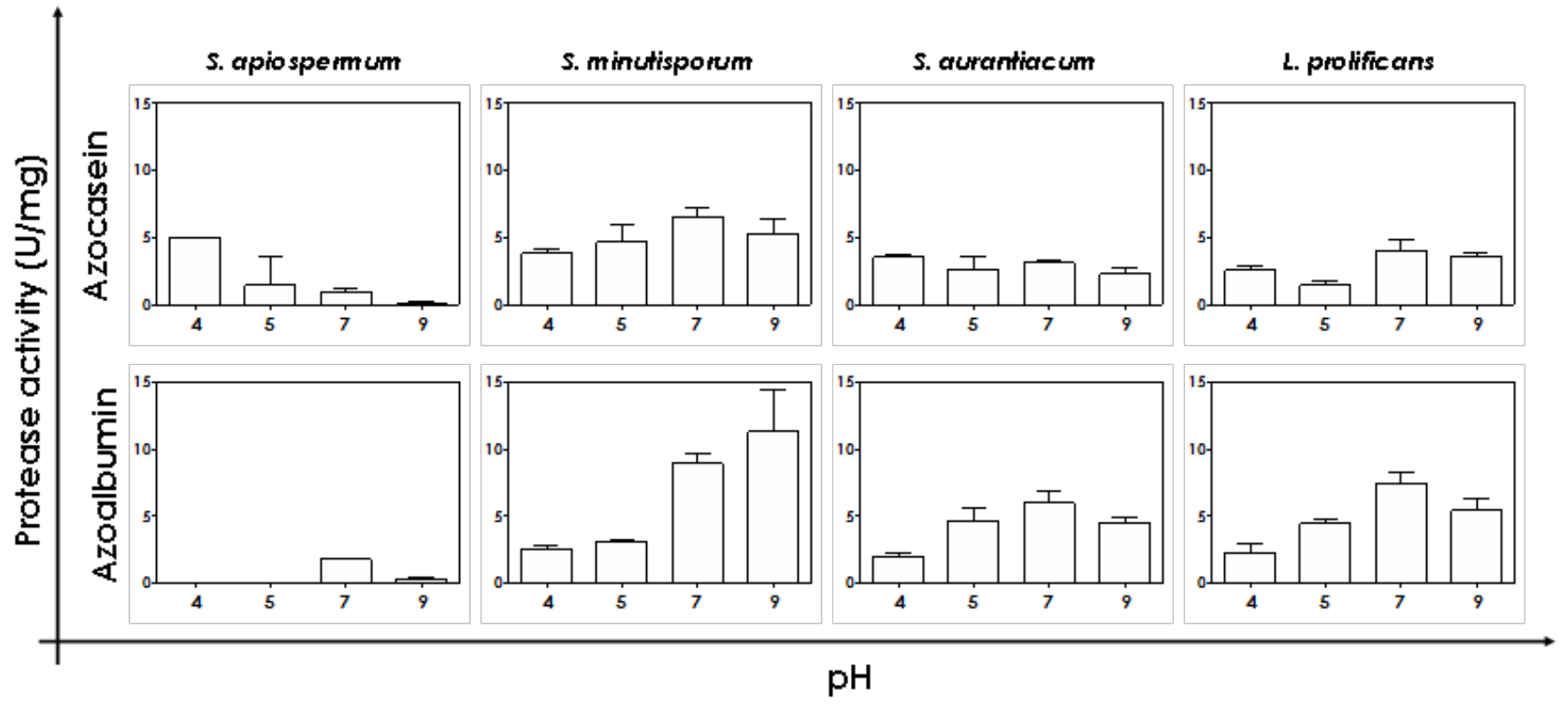

Figure 2 Protease activity measured in the 72 hours-biofilm-derived supernatants from S. apiospermum, S. minutisporum, S. aurantiacum and L. prolificans. The enzymatic activities were measured after I hour at $37^{\circ} \mathrm{C}$ using different $\mathrm{pH}$ values and two distinct proteinaceous substrates, azocasein and azoalbumin. The protease activity was expressed as arbitrary units $(U)$ per milligram of protein, where one unit of activity is equivalent to the variation of $0.00 \mathrm{I} n \mathrm{~m}$ in the optical density per $\mathrm{min}$ at $366 \mathrm{~nm}$. The bars represent the means \pm standard deviations from at least three independent experiments.
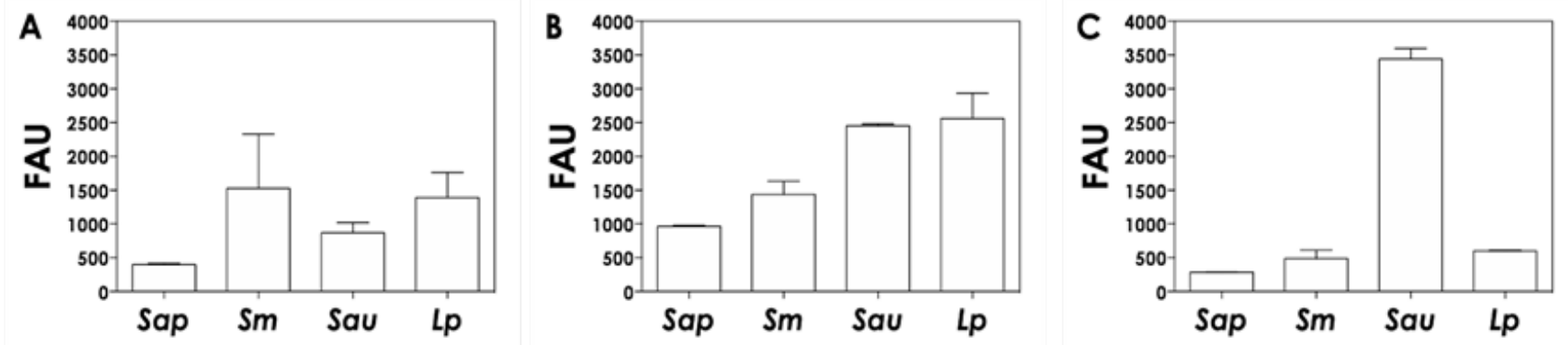

Figure 3 Lipase activity measured in the 72 hours-biofilm-derived supernatants from S. apiospermum (Sap), S. minutisporum (Sm), S. aurantiacum (Sau) and L prolificans (Lp). The enzymatic activities were measured after I hour at $37^{\circ} \mathrm{C}$ at, $\mathrm{pH} 8.0$, using three distinct lipid substrates: (A) 4-methylumbelliferyl butyrate, (B) 4-methylumbelliferyl heptanoate and (C) 4-methylumbelliferyl oleate. The lipase activity was expressed as fluorescence arbitrary units (FAU). The bars represent the means \pm standard deviations from at least three independent experiments.

\section{Discussion}

As previously reported by our research group, Scedosporium/ Lomentospora species form mature biofilms after 72 hours of incubation on several abiotic and biotic surfaces.$^{5,6} \mathrm{In}$ the present work, we described that those biofilm-forming cells were able to release both lipases and proteases. The role of secreted hydrolytic enzymes in mature biofilms has been linked to crucial biological events, such as nutrient acquisition and cell dispersion, in several fungal species, including Candida albicans and Trichosporon inkin. ${ }^{7-9}$ The dispersion of cells confined in a mature biofilm structure is a relevant event on microbial transmission from environmental reservoirs to human hosts as well as in dissemination of focal infection within the host. ${ }^{9}$ The secreted hydrolytic enzymes cleave the biofilm extrapolymeric matrix, which acts like a glue holding the biomass together, releasing microbial cells into the environment that permit the colonization of other environments. The expression of secreted aspartic proteases (SAPs), phospholipase B (PLB) and lipase (LIP) genes in C. albicans is directly associated with biofilm growth on mucosal surfaces as well in abiotic surfaces. ${ }^{10}$ In addition, sessile $C$. albicans cells secrete more aspartic proteases than planktonic cells, ${ }^{7}$ demonstrating the importance of secretion of hydrolytic enzymes on biofilm formation and maturation.

\section{Conclusion}

Biofilm-growing cells of Scedosporium and Lomentospora species extracellularly released hydrolytic enzymes, including proteases (with the ability to cleave distinct proteinaceous substrates at wide range of $\mathrm{pH}$ ) and lipases (capable of cleaving molecules with different lipid chain sizes), which can contribute with the physiological homeostasis of the biofilm structure. 


\section{Funding information}

This work was supported by grants from Fundação Carlos Chagas Filho de Amparo à Pesquisa do Estado do Rio de Janeiro (FAPERJ), Conselho Nacional de Desenvolvimento Científico e Tecnológico (CNPq) and Coordenação de Aperfeiçoamento de Pessoal de Nível Superior (CAPES - Financial code 001).

\section{Acknowledgments}

The authors would like to thank Denise Rocha de Souza, supported by FAPERJ scholarships, for her technical assistance.

\section{Conflicts of interest}

The authors declare that there is no conflict of interest.

\section{References}

1. Santos ALS, Mello TP, Souza LR, et al. Biofilm: a robust and efficient barrier to antifungal chemotherapy. J Antimicrobial Agents. 2015;1:1-4.

2. Mello TP, Bittencourt VCB, Liporagi-Lopes LC, et al. Insights into the social life and obscure side of Scedosporium/Lomentospora species: ubiquitous, emerging and multidrug-resistant opportunistic pathogens. Fungal Biol Rev. 2019;33(1):16-46.

3. Silva BA, Pinto MR, Soares RMA, et al. Pseudallescheria boydii releases metallopeptidases capable of cleaving several proteinaceous compounds. Res Microbiol. 2006;157(5):425-432.
4. Han Z, Kautto L, Nevalainen H. Secretion of proteases by an opportunistic fungal pathogen Scedosporium aurantiacum. PLoS One. 2017;12(1):1-19.

5. Mello TP, Aor AC, Gonçalves DS, et al. Assessment of biofilm formation by Scedosporium apiospermum, S. aurantiacum, S. minutisporum and Lomentospora prolificans. Biofouling. 2016;32(7):737-749.

6. Mello TP, Oliveira SSC, Frasés S, et al. Surface properties, adhesion and biofilm formation on different surfaces by Scedosporium spp. and Lomentospora prolificans. Biofouling. 2018;34(7):800-814.

7. Mendes A, Mores AU, Carvalho AP, et al. Candida albicans biofilms produce more secreted aspartyl protease than the planktonic cells. Biol Pharm Bull. 2007;30(9):1813-1815.

8. Kaplan JB. Biofilm dispersal: mechanisms, clinical Implications, and potential therapeutic uses. J Dent Res. 2010;89(3):205-218.

9. Cordeiro RA, Serpa R, Alexandre CFU, et al. Trichosporon inkin biofilms produce extracellular proteases and exhibit resistance to antifungals. $J$ Med Microbiol. 2015; 64(11):1277-1286.

10. Nailis H, Kucharíková S, Ricicová M, et al. Real-time PCR expression profiling of genes encoding potential virulence factors in Candida albicans biofilms: identifications of model-dependent and -independent gene expression. BMC Microbiol. 2010;10:114. 